\title{
Inpatient Point-of-Care HIV Early Infant Diagnosis in Mozambique to Improve Case Identification and Linkage to Antiretroviral Therapy
}

Mércia Matsinhe, ${ }^{a, b}$ Timothy Bollinger, ${ }^{c}$ Nilza Lee, ${ }^{d}$ Osvaldo Loquiha, ${ }^{c}$ Bindiya Meggi, ${ }^{b}$ Nédio Mabunda, Chishamiso Mudenyanga, ${ }^{C}$ Dadirayi Mutsaka, ${ }^{c}$ Marcelina Florêncio, ${ }^{a}$ Aurora Mucaringua, ${ }^{d}$ Eugénia Macassa, ${ }^{\text {a Amir Seni, }}{ }^{d}$ llesh Jani, ${ }^{b}$ W. Chris Buck ${ }^{e}$

\section{Key Findings}

- Monthly virologic testing volume in HIV-exposed infants increased $97 \%$ with introduction of point-ofcare early infant diagnosis testing.

- There was a $29.7 \%$ positivity rate for inpatient point-ofcare tests performed.

- Antiretroviral therapy initiation for infants with positive tests improved $64 \%$ with introduction of point-of-care early infant diagnosis.

\section{Key Implications}

- Pediatric inpatient wards are high-yield sites for HIV case identification, and inpatient early infant diagnosis is an important backstop to outpatient follow-up of exposed infants for prevention of mother-to-child transmission.

- Point-of-care testing improves inpatient early infant diagnosis performance, facilitating more timely initiation of antiretroviral in HIV-infected infants, and point-of-care early infant diagnosis expansion plans should prioritize high-volume pediatric wards.

\footnotetext{
a Hospital Central de Maputo, Maputo, Mozambique.

b Instituto Nacional de Saúde, Maputo, Mozambique.

c Clinton Health Access Initiative, Maputo, Mozambique.

${ }^{d}$ Hospital Central de Beira, Beira, Mozambique.

e University of California Los Angeles, David Geffen School of Medicine, Los Angeles, USA.

Correspondence to W. Chris Buck (wbuck@mednet.ucla.edu).
}

\section{ABSTRACT}

Introduction: Novel approaches to case identification and linkage to antiretroviral therapy (ART) are needed to close gaps in early infant diagnosis (EID) of HIV. Point-of-care (POC) EID is a recent innovation that eliminates the long turnaround times of conventional EID that limit patient management in the inpatient setting. The initial deployment of POC EID in Mozambique focused primarily on outpatient clinics; however, 2 high-volume tier-4 pediatric referral hospitals were also included.

Methods: To assess the impact of inpatient POC EID, a retrospective review of testing and care data from Hospital Central de Beira (HCB) and Hospital Central de Maputo (HCM) was performed for the period September 2017 to July 2018, with comparison to the 8-month pre-POC period when dried blood spots were used for conventional EID.

Results: Monthly testing volume increased from 8.5 tests pre-POC to 17.6 tests with $P O C(P<.001)$. Among 511 children with $P O C$ testing, the median age was 5 months, there was ongoing breasffeeding in $326(63.8 \%)$, and $136(26.6 \%)$ of mothers and $146(28.6 \%)$ of infants had not received ART or antiretroviral prophylaxis, respectively. POC tests were positive in $152(29.7 \%)$ infants, and 52 (37.5\%) had a previous negative DNA polymerase chain reaction through the conventional outpatient EID program. Linkage to ART for infants with HIV-positive tests improved $64 \%$ during the POC period $(P=.002)$. Inpatient mortality for infected infants during the POC period was $28.2 \%$. Excluding these deaths, $61.2 \%$ of eligible infants initiated ART as inpatients, but only $29.8 \%$ of those discharged without ART were confirmed to have initiated as outpatients.

Conclusions: Inpatient wards are a high-yield site for EID and ART initiation that have historically been overlooked in programming for prevention of mother-to-child transmission. POC platforms represent a transformative opportunity to increase inpatient testing, make definitive diagnoses, and improve timely linkage to ART. Scale-up plans should prioritize pediatric wards.

\section{INTRODUCTION}

D espite massive scale-up over the past decade, pediatric antiretroviral therapy (ART) coverage rates in sub-Saharan Africa remain low. In Mozambique, only an estimated $50 \%$ of HIV-infected children were on ART at the end of 2018, compared with a 55\% coverage rate in adults. ${ }^{1,2}$ Low pediatric coverage rates can, in part, be attributed to significant challenges with retention of 


Conventional EID
programs do not
adequately serve
pediatric inpatient
wards and
represent a
missed
opportunity to
reduce the impact
of HIV in children.

mother-infant pairs in prevention of mother-tochild transmission (PMTCT) services with only $62 \%$ of exposed infants of women living with HIV enrolled in antenatal care having an early infant diagnosis (EID) virologic test by 2 months of age in 2018. ${ }^{1}$ For HIV-exposed infants (HEI) retained in care, the complexity of establishing the HIV status of children under 18 months of age likely also contributes to low pediatric ART coverage. Definitive diagnosis in HEIs requires a virologic EID test, which until recently was only available in centralized reference laboratories, with delayed result delivery due to transport times and the need to process samples from many health facilities. ${ }^{3}$ Slow delivery of EID results and subsequent delayed ART initiation lead to higher mortality rates among HIVinfected children. ${ }^{4}$

Point-of-care (POC) testing for EID is a recent innovation that permits health care systems to decentralize testing and bypass the inefficient networks needed for centralized testing platforms. ${ }^{3}$ POC EID has well-documented impact and success, virtually eliminating turnaround times and therefore permitting a same-day testing and treatment paradigm for HIV-infected infants. ${ }^{5-8}$ In Mozambique, a cluster randomized trial showed that $90 \%$ of participants accessing POC EID were linked to timely ART compared with $13 \%$ in the standard of care. ${ }^{9}$

POC EID testing in Mozambique uses the Abbott mPIMA platform, which gives results in approximately 1 hour, and was scaled up from 2017 and 2018 in a strategic rollout that prioritized deployment to high-volume outpatient services at public primary health care facilities, consistent with international implementation recommendations. ${ }^{10,11}$ In recognition of the opportunity to also reach hospitalized infants who are more likely to have advanced HIV and need urgent ART to prevent mortality, pediatric wards in 2 large referral hospitals, Hospital Central de Maputo (HCM) and Hospital Central de Beira (HCB), were included in the implementation plan. Despite their perceived ease of access to reference laboratories with conventional molecular testing equipment, inpatient wards in hospitals in Mozambique historically face similar long turnaround times for EID results compared with outpatient health facilities, with an average of 36 days for the 11 largest hospitals in the country in 2016 compared with 47 days for health centers. ${ }^{12}$ These settings represent considerable wastage of conventional EID resources because patient discharge usually occurs before results are available, and post-discharge follow-up is complicated by long distances between patients' homes and the referral hospital. Furthermore, the national EID program does not use a unique patient identifier to facilitate tracking results across health facilities. As such, conventional EID programs do not adequately serve pediatric inpatient wards and represent a missed opportunity, especially since these settings are known to have high yields of HIV-positive tests when EID testing is part of provider-initiated testing and counseling (PITC) is operationalized. ${ }^{13-17}$

We conducted an early assessment of the impact of POC EID deployment in the pediatric wards of HCM and HCB.

\section{METHODS}

\section{Study Design, Setting, and Participants}

This study was a retrospective review of routine EID testing and patient care data from HCM and $\mathrm{HCB}$, tier-4 reference hospitals providing the highest level of care in the public sector in the southern and central regions of Mozambique, respectively. In 2018, HCM admitted an average of 866 children per month, and HCB admitted an average 536 children per month. In both hospitals, the median age of admission is below 2 years old. The POC study period began from the time of POC implementation at each site (February 2017 for HCB and September 2017 for HCM) through July 2018. Eight months of pre-POC data, when testing was performed via dried blood spots (DBSs), were included from each site for comparison. Per national guidelines, HEIs aged 1 to 18 months who underwent inpatient EID testing were included. ${ }^{18}$ Patients with nonvalid EID results and those referred from other health facilities for EID testing (i.e., infants not admitted to the hospital but accessing the POC EID platform) were excluded. Patients who were known to be HIV infected at the time of admission were not eligible for EID testing and were not included.

\section{National PITC and EID Guidelines}

Infants already known to be HIV exposed at the time of admission (identified through review of maternal and child health documents and caregiver medical history) were routinely offered initial EID testing if standard testing at 1 month of age had been missed. They were also generally offered repeat EID testing if they had outdated previous negative outpatient EID test results or presenting conditions suggestive of HIV infection. For infants whose mothers had unknown serostatus or whose last negative test was more than 3 months prior to admission, national policy was to conduct routine 
opt-out ward-based PITC of mothers using rapid antibody tests to newly identify HEIs eligible for EID testing. ${ }^{18}$ The national EID algorithm during the time period of this study called for virologic testing at enrollment in the HEI clinic (recommended at 1 month of age), at 9 months if rapid antibody testing was positive, and at any time infants had signs and symptoms suggestive of HIV infection. For inpatient EID, guidelines are not specific about when to repeat virologic testing for HEIs who previously tested negative, but generally speaking, EID testing is repeated in children with malnutrition, developmental delay, or infectious illness that could be associated with immune suppression, or if previous testing was more than 2 months prior to admission. Active phone tracing of infected patients identified through hospitalbased EID to confirm their continuity of care after discharge to primary health centers was recommended, but it was more routinely done at HCM than HCB during the period of this study.

\section{Data Collection}

For the pre-POC period, DBS EID data were collected for each site from a national EID data database that contains test results, demographic information, and clinical information from standard national EID requisition forms which include PMTCT information on maternal ART, infant prophylaxis, breastfeeding status, and previous EID testing. ART information for the pre-POC period was obtained from site ART registers. A more comprehensive set of data were collected for the POC EID period from sources including onsite EID logbooks, EID requisition forms, 2 web-based databases (POC connectivity and the national online EID portal), ART registers, and call logs from a patient followup program (HCM only). A trained team of data collectors reviewed all available data sources to populate an anonymous, structured database that recorded patient demographics, EID testing dates and results, ART information, and follow-up status for all patients with POC EID testing in the study period.

\section{Data Analysis}

Data were collected and organized into Microsoft Excel (2003), and data analysis was conducted using STATA v12 (StataCorp@, 2011). Descriptive summary statistics were produced for testing volumes and positivity rates. Chi-square and Fisher's exact tests were applied to investigate differences in preand post-POC implementation results in addition to POC positivity rates for a set of patient characteristics including age, sex, PMTCT regimens, and previous access to conventional DNA polymerase chain reaction (PCR) results. All statistical analyses used $P$ values and 5\% significance level for inference.

\section{Ethical Considerations}

Ethical approval for this assessment was obtained from Mozambique's National Health Bioethics Committee reference 80/CNBS/14. The Scientific Directorates of HCM and HCB also approved the study. These boards did not require the study to obtain consent from caregivers for use of the routine EID and ART data included in the analysis.

\section{RESULTS}

\section{POC Study Population Characteristics}

A total of 511 HEIs were tested with POC at both hospitals during the study period (330 patients over 18 months at $\mathrm{HCB}$, and 181 patients over 11 months at HCM). The median age was 5 months, and 232 patients $(45.4 \%)$ were girls. A previous negative DNA PCR performed at the primary health facility level of care as part of routine EID was documented for 219 (42.9\%) infants. No history of antiretroviral prophylaxis or ART for PMTCT was available for 136 (26.6\%) of mothers, 146 (28.6\%) of infants had not received nevirapine prophylaxis, and $326(63.8 \%)$ of infants were still being breastfed (Table 1).

\section{POC Test Results and ART Initiation}

POC tests were positive in 152 (29.7\%) of the HEIs tested. Of these HIV positive infants, 74 (48.7\%) initiated ART during their hospitalization. A total of 43 inpatient deaths $(28.2 \%)$ occurred among infants with confirmed HIV infection, including 12 who initiated ART in the hospital. Excluding the 31 infants who died in the hospital prior to ART initiation, $61.2 \%(74 / 121)$ of eligible patients initiated ART while admitted. For the 109 infected infants who were discharged, 66.1\% (41/62) of those who initiated ART as inpatients were confirmed to be active on ART on follow-up, and only $29.8 \%$ (14/47) of those who did not initiate ART as inpatients were confirmed to be active on ART (Figure).

\section{Clinical and Demographic Variables Associated With POC Positivity}

Infants whose mothers were on ART had a $27.4 \%$ positivity rate compared with $38.2 \%$ when there was no maternal ART $(P=.047)$. Infants who had received nevirapine prophylaxis had a 
TABLE 1. Clinical and Demographic Characteristics of HIV-Exposed Infants in Point-of-Care Early Infant Diagnosis Study From Inpatient Wards of 2 Pediatric Referral Hospitals, Mozambique

\begin{tabular}{|c|c|c|c|}
\hline \multirow[b]{2}{*}{ Variable } & \multicolumn{3}{|c|}{ Patients, $n$ (\%) } \\
\hline & HCB & HCM & Total \\
\hline \multicolumn{4}{|l|}{ Age, months } \\
\hline $1-2$ & $101(30.6)$ & 45 (24.9) & $146(28.6)$ \\
\hline $3-5$ & $67(20.3)$ & $54(29.8)$ & $121(23.7)$ \\
\hline $6-8$ & $73(22.1)$ & 33 (18.2) & 106 (20.7) \\
\hline $9-11$ & $40(12.1)$ & 27 (14.9) & $67(13.1)$ \\
\hline$\geq 12$ & 48 (14.5) & $20(11.0)$ & $68(13.3)$ \\
\hline Missing & $1(0.3)$ & $2(1.1)$ & $3(0.6)$ \\
\hline \multicolumn{4}{|l|}{ Sex } \\
\hline Female & $148(44.8)$ & $84(46.4)$ & 232 (45.4) \\
\hline Male & $182(55.2)$ & $97(53.6)$ & $279(54.6)$ \\
\hline \multicolumn{4}{|l|}{ Previous DNA PCR } \\
\hline Yes & $138(41.8)$ & $81(45.0)$ & $219(42.9)$ \\
\hline $\mathrm{No}^{\mathrm{a}}$ & $192(58.2)$ & $99(55.0)$ & $292(57.1)$ \\
\hline \multicolumn{4}{|l|}{ Maternal PMTCT } \\
\hline Prophylaxis & $2(0.6)$ & $4(2.2)$ & $6(1.2)$ \\
\hline Full ART & $210(63.6)$ & $133(73.5)$ & $343(67.1)$ \\
\hline None & $105(31.8)$ & $31(17.1)$ & $136(26.6)$ \\
\hline Missing & $13(3.9)$ & $13(7.2)$ & $26(5.1)$ \\
\hline \multicolumn{4}{|l|}{ Infant PMTCT } \\
\hline NVP prophylaxis & $207(62.7)$ & $130(71.8)$ & $337(65.9)$ \\
\hline None & $113(34.2)$ & 33 (18.2) & $146(28.6)$ \\
\hline Missing & $10(3.0)$ & $18(9.9)$ & $28(5.5)$ \\
\hline \multicolumn{4}{|l|}{ Current breastfeeding } \\
\hline Yes & $233(70.6)$ & $93(51.4)$ & $326(63.8)$ \\
\hline No & $78(23.6)$ & $73(40.3)$ & $151(29.5)$ \\
\hline Missing & $19(5.8)$ & $15(8.3)$ & $34(6.7)$ \\
\hline Total & $330(100)$ & $180(100)$ & $511(100)$ \\
\hline
\end{tabular}

Abbreviations: ART, antiretroviral therapy; HCB, Hospital Central de Beira; HCM, Hospital Central de Maputo; NVP, nevirapine; PCR, polymerase chain reaction; PMTCT, prevention of mother-to-child transmission.

a Includes infants with unknown or undocumented previous testing.

$26.4 \%$ positivity rate compared to $37.0 \%$ in those who had not $(P=.018)$. Infants who were still breastfeeding at the time of testing had a $33.1 \%$ positivity rate compared with $22.5 \%$ in those who were not $(P=.019)$. No significant differences in positivity were found based on age, sex, and previous negative DNA PCR (Table 2). Of children who tested positive,
$37.5 \%(52 / 152)$ had a documented previous negative DNA PCR test performed prior to admission.

\section{POC Positivity by Ward}

Significant differences in POC test positivity were found based on the ward from which patients were referred for inpatient testing, with malnutrition 
FIGURE. Point-of-Care Early Infant Diagnosis Testing and Linkage to Antiretroviral Therapy, Among HIVExposed Infants From Two Pediatric Referral Hospitals, Mozambique

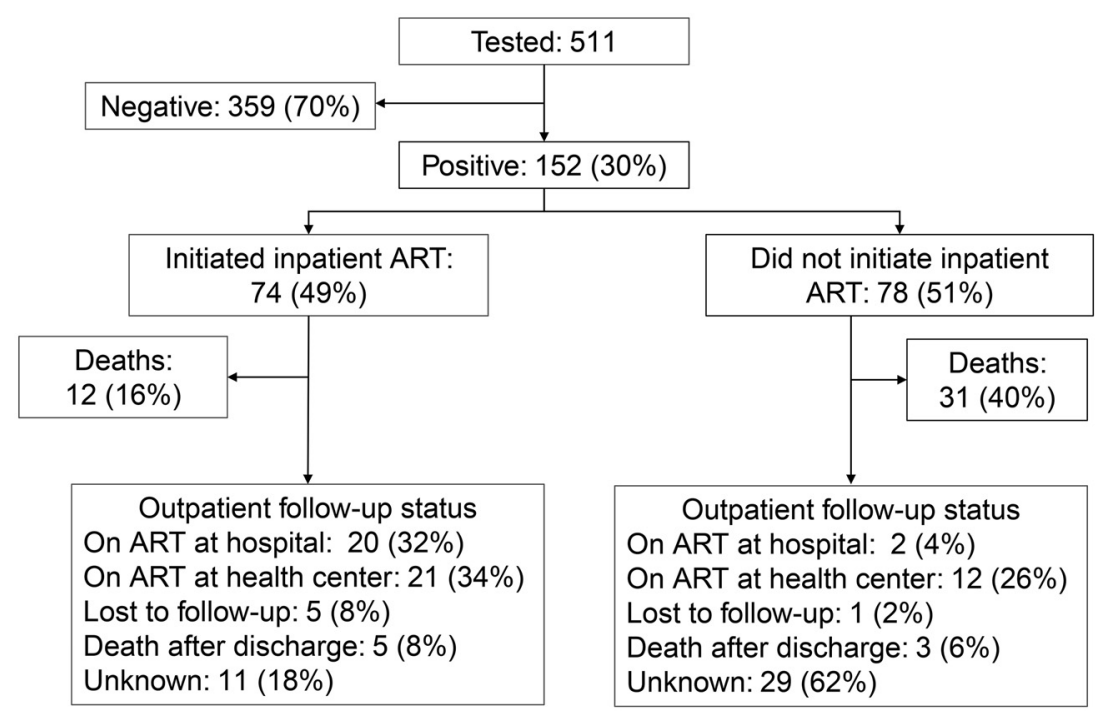

Abbreviation: ART, antiretroviral therapy.

$(41.1 \%)$, the pediatric intensive care unit $(40.0 \%)$, and the breastfeeding ward $(38.3 \%)$ having the highest rates (Table 3).

\section{Utilization, Positivity, and Linkage to ART Compared With Pre-POC DBS Testing}

In combined analysis from both hospitals, testing volume increased $97 \%$ from an average of 8.9 inpatient tests per month with DBSs in the pre-POC period to 17.6 tests per month with POC $(P<.001)$. The median age of tested infants was 4 months for the pre-POC period and 5 months for the POC period. Test positivity decreased from $45.5 \%$ pre-POC to $29.7 \%$ with POC $(P<.001)$. Documentation of successful linkage to ART (inpatient or outpatient) increased from $35.4 \%$ pre-POC to $57.9 \%$ with POC $(P=.002)$.

\section{DISCUSSION}

This study shows that inpatient POC EID is both feasible and effective. Not only did the placement of POC technology in these hospital settings increase overall testing volume and newly identify a high number of children with HIV-positive tests, but the immediate onsite POC test results also permitted much improved linkage to ART for these children compared with the pre-POC period.
A highly significant $97 \%$ increase in testing volume per month occurred after allocation of POC, which we believe is best explained by clinicians being more likely to order EID testing when timely results were available. Improved inpatient PITC at both hospitals during the time period of the study could have contributed to more newly identified HEIs, but PITC testing data were not included in this study and the large majority of EID tests are performed in infants already known to be HIV exposed. No dramatic changes in maternal antenatal HIV prevalence, annual admission volumes, or EID eligibility criteria occurred during the time period of the study that would otherwise explain this large increase in testing volume. Yet, despite the large increase in testing volume, the POC platforms still may have been underutilized. This circumstance is of particular concern at HCM, which is in a higher-HIV prevalence province and has approximately $60 \%$ more admissions per month, but had fewer inpatient POC tests performed per month than did HCB (16.5 versus 18.3). ${ }^{19}$ Increased POC EID testing is needed in wards that admit HEIs 12-18 months of age, which represented only $36.6 \%$ of the test requests in this study. This finding is important in light of new EID algorithm recommendations from the World Health Organization that call for virologic

\author{
The immediate \\ onsite POC test \\ results permitted \\ much improved \\ linkage to ART for \\ children with HIV \\ infection \\ compared with the \\ pre-POC period.
}


TABLE 2. Point-of-Care Early Infant Diagnosis Positivity by Clinical and Demographic Variables Among HIVExposed Infants From Inpatient Wards of 2 Pediatric Referral Hospitals, Mozambique

\begin{tabular}{|c|c|c|c|}
\hline Variable & Negative Test, $n$ (\%) & Positive Test, $\boldsymbol{n}(\%)$ & Chi-Square $P$ Value \\
\hline \multicolumn{4}{|l|}{ Age, months } \\
\hline $1-2$ & $113(77.4)$ & $33(22.6)$ & \multirow{6}{*}{0.127} \\
\hline $3-5$ & $77(63.6)$ & $44(36.4)$ & \\
\hline $6-8$ & $70(66.0)$ & $36(34.0)$ & \\
\hline $9-11$ & $48(71.6)$ & $19(28.4)$ & \\
\hline$\geq 12$ & $49(72.1)$ & $19(27.9)$ & \\
\hline Missing & $2(66.7)$ & $1(33.3)$ & \\
\hline \multicolumn{4}{|l|}{ Sex } \\
\hline Female & $160(69.0)$ & $72(31.0)$ & \multirow[b]{2}{*}{0.561} \\
\hline Male & 199 (71.3) & $80(28.7)$ & \\
\hline \multicolumn{4}{|l|}{ Previous DNA PCR } \\
\hline Yes & $162(74.0)$ & $57(26.0)$ & \multirow{2}{*}{0.106} \\
\hline No & $196(67.4)$ & $95(32.6)$ & \\
\hline \multicolumn{4}{|l|}{ Maternal PMTCT } \\
\hline Prophylaxis & $5(83.3)$ & $1(16.7)$ & \multirow{4}{*}{$0.047^{a}$} \\
\hline Full ART & $249(72.6)$ & $94(27.4)$ & \\
\hline None & $84(61.8)$ & $52(38.2)$ & \\
\hline Missing & $21(80.8)$ & $5(19.2)$ & \\
\hline \multicolumn{4}{|l|}{ Infant PMTCT } \\
\hline NVP prophylaxis & $248(73.6)$ & $89(26.4)$ & \multirow{3}{*}{0.018} \\
\hline None & $92(63.0)$ & $54(37.0)$ & \\
\hline Missing & $19(67.9)$ & $9(32.1)$ & \\
\hline \multicolumn{4}{|l|}{ Current breastfeeding } \\
\hline Yes & $218(66.9)$ & $108(33.1)$ & \multirow{3}{*}{0.019} \\
\hline No & $117(77.5)$ & $34(22.5)$ & \\
\hline Missing & $24(70.6)$ & $10(29.4)$ & \\
\hline Total & $359(70.3)$ & $152(29.7)$ & \\
\hline
\end{tabular}

Abbreviations: ART, antiretroviral therapy; NVP, nevirapine; PCR, polymerase chain reaction; PMTCT, prevention of mother to child transmission.

a Fisher's exact test.

testing in HEIs $>9$ months of age given the possibility of false-negative rapid HIV test results. ${ }^{1,20-22}$

Concerningly, a study of pediatric inpatient PITC from wards in Mozambique reported that coverage rates are low. ${ }^{13}$ Routine opt-out rapid antibody testing of breastfeeding mothers is a crucial first step to newly identify HEIs who need EID testing, and this step can help improve POC EID platform utilization. The same study also showed that hospitals that relied on DBSs for EID often did not test or retest exposed infants and presumptive HIV diagnosis was rarely made. ${ }^{13}$ POC platforms bypass the inherent delays that come with DBS-based EID; eliminate the need for inpatient presumptive HIV diagnosis, which has been consistently underutilized; and have the potential to strengthen pediatric inpatient PITC and improve case detection. Inpatient POC EID can also serve as an important backstop to traditional outpatient HEI-clinic EID, as $37.5 \%$ of the infants with 
TABLE 3. Point-of-Care Early Infant Diagnosis Positivity Among HIV-Exposed Infants From Inpatient Wards of 2 Pediatric Referral Hospitals, Mozambique, by Referral Ward

\begin{tabular}{|c|c|c|c|}
\hline Ward & Negative Test, $\boldsymbol{n}(\%)$ & Positive Test, n (\%) & Fisher's Exact Test $P$ Value \\
\hline Nursery & $12(100.0)$ & $0(0)$ & \multirow{9}{*}{0.002} \\
\hline Breastfeeding $^{a}$ & $108(61.7)$ & $67(38.3)$ & \\
\hline General ward $^{b}$ & $32(82.1)$ & $7(17.9)$ & \\
\hline Infectious diseases & $130(74.7)$ & $44(25.3)$ & \\
\hline Malnutrition & $33(58.9)$ & $23(41.1)$ & \\
\hline Pediatric intensive care unit & $15(60.0)$ & $10(40.0)$ & \\
\hline Other ${ }^{c}$ & $5(83.3)$ & $1(16.7)$ & \\
\hline Missing & $24(100.0)$ & $0(0)$ & \\
\hline Total & $359(70.3)$ & $152(29.7)$ & \\
\hline
\end{tabular}

positive POC tests had a previous negative outpatient PCR and were presumably infected during breastfeeding.

The $29.7 \%$ overall positivity rate for POC EID from this study is significantly higher than other results reported from the region. A POC EID evaluation from 8 African countries reported a $15.2 \%$ prevalence in pediatric inpatients (hospitalized children represented only $2.9 \%$ of the total cohort), and a study from Malawi, where $48.9 \%$ of the infants tested with POC came from inpatient wards, had an overall positivity rate of $5.7 \%$ (no inpatient/outpatient disaggregation provided). ${ }^{5,7}$

The EID positivity rate was even higher before POC implementation, at $45.5 \%$. Although some of this difference could be explained by improved PMTCT coverage in the later POC period, it is also likely that DBS testing was only performed in HEIs with more advanced signs and symptoms of HIV infection. ${ }^{1}$ Not all admitted HEIs need repeat virologic testing with POC, but the ease of use and timely results seem to lower the clinical threshold for testing to include more subtle early signs of infection, thereby allowing for more timely diagnosis of HIV with better treatment outcomes.

The PMTCT program in Mozambique continues to struggle to reduce vertical transmission, with an estimated rate of $15 \%$ (much of which occurs via breastfeeding), challenges with maternal ART adherence and retention, and high rates of maternal seroconversion during pregnancy and lactation..$^{23-26}$ Undiagnosed infants will often become ill and require hospitalization, and this study showed the high toll of such late presentation in terms of inpatient mortality at $28.2 \%$. This rate was higher than the $22 \%$ mortality rate reported in a recent pediatric inpatient study from Kenya that included infants and older children, and it highlights the importance of strengthening serial maternal HIV testing throughout pregnancy and the breastfeeding period, as well as outpatient EID, so diagnoses can be made before children develop advanced disease requiring hospitalization. $^{27}$

Confirmed ART initiation for infants with positive inpatient EID tests was $64 \%$ higher in the POC period, facilitated by same-day actionable results. Despite previous efforts to prioritize central laboratory testing of DBS samples from inpatient wards, the turnaround times for conventional DNA PCR (36-day average for the 11 largest hospitals in Mozambique in 2016) meant that most hospitalized patients had been discharged before their results were returned, despite being expedited. ${ }^{12}$ Furthermore, posthospitalization follow-up often occurs at primary health care centers closer to patients' homes, and the resources needed for routine active tracing of infected infants via phone or home visits are generally lacking. Consequently, these conventional results never reached the children's caregivers, contributing to reduced linkage to ART. This study shows that such hurdles to timely ART initiation for infants with HIV infection

\section{The PMTCT program in Mozambique struggles to reduce vertical transmission.}


diagnosed during hospitalization can be addressed with the use of POC EID.

With the exclusion of infants who died during hospitalization prior to ART initiation, $61.2 \%$ of those with positive tests initiated as inpatients. Our data set did not allow for analysis of why some infants did not initiate ART as inpatients, but anecdotally the most common reason was inpatient tuberculosis diagnosis with guidelines recommending 2 weeks of treatment before ART should be started. An additional $29.8 \%$ of HIVinfected infants who were discharged without ART were confirmed to have initiated as outpatients. These numbers compare unfavorably to other POC studies, which reported ART linkage rates of $86.3 \%$ and $91 \%$, but did not disaggregate inpatient versus outpatient timing. ${ }^{5,7}$ A large difference was found in confirmed ART post discharge in patients who initiated as inpatients versus those who did not ( $66.1 \%$ vs. $29.8 \%)$, suggesting that an ideal model would promote ART initiation during admission to the extent possible. A recent clinical trial showed no early mortality benefit to urgent inpatient ART initiation in children, but the findings from this study suggest that initiation after stabilization but before discharge may help improve access to and retention on ART. ${ }^{27}$ A clear need exists to improve active follow-up of infants diagnosed during hospitalization at these sites to ensure successful linkage to ongoing ART.

The results from this study suggest that POC EID can further improve access to EID and pediatric ART by broadening the considerations for potential placement scenarios. Our findings suggest there is clear benefit to deploying POC testing to hospitals with subsequent establishment of contextappropriate ART initiation models that readily decentralize back to primary care. However, this consideration for further deployment of POC testing to inpatient settings does not need to be limited to large pediatric hospitals; the dynamics of inpatient reality (late presentation, incomplete EID testing history, admission duration shorter than conventional DNA PCR turnaround times) are applicable to smaller wards throughout the country whose patients would also benefit from inpatient POC EID. The ability to now multiplex on POC instruments, such as the HIV viral load assays that are now also being performed at HCM and HCB on the Abbott mPIMA analyzer used in this study, the advent of birth EID testing opportunities, and combined inpatient/outpatient testing at sites with both EID clinics and pediatric wards can generate testing volumes that justify deployment to settings such as district hospitals that previously may not have met POC allocation criteria. Indeed, there remain deployment opportunities to further reach HEIs across the health care system, and POC consistently proves to be an effective and feasible solution-one that will be needed to help reach national and global targets.

\section{Limitations}

This study had limitations that need mention. A smaller set of data variables was available for the pre-POC period, and we were not able to perform a comparison of hospital outcomes from before and after the implementation of inpatient POC EID. The number of HIV-exposed children who were admitted and would need EID testing during the pre-POC and POC periods was also not part of the data available for this study, so our conclusions about availability of POC driving increased testing demand come with qualifications. Phone followup to verify outpatient ART status did not occur at a standard time interval post hospital discharge, and systems were lacking for timely and routine phone follow-up of discharged patients at HCB, possibly leading to an underestimation of ART linkage. Given the retrospective methodology, several clinical and demographic variables had missing data that could not be captured. The data presented here are from a 2017-2018 period, but the results reflect the inpatient EID realities in Mozambique and are useful for ongoing programmatic planning.

\section{- CONCLUSIONS}

Inpatient wards are a high-yield site for case identification and ART initiation that have historically been overlooked in PMTCT programming. POC platforms can increase inpatient EID testing volume and represent a transformative opportunity to improve the diagnosis and treatment of HIV in hospitalized infants. POC EID scale-up plans should include pediatric wards as priority sites for future expansion.

Acknowledgments: We would like to recognize the medical and nursing teams at Hospital Central de Maputo (HCM) and Hospital Central de Beira (HCB), who provided care to the children included in this study. We also acknowledge the contributions of other staff from the Mozambique Ministry of Health, Instituto Nacional de Saúde, and Clinton Health Access Initiative who provided key assistance to the implementation of point-of-care early infant diagnosis at $\mathrm{HCB}$ and $\mathrm{HCM}$ with funding from Unitaid and United Nations Children's Fund.

Author Contributions: $M M, N L$, and WCB contributed to study design, data collection, analysis, and manuscript drafting. WCB led manuscript revision. TB contributed to study design, analysis, and manuscript drafting. OL had principal responsibility for data analysis and contributed 
to manuscript revisions. BM, NM, CM, DM, EM, AS, and IJ contributed to study design and manuscript revision. MF and $A M$ contributed to data collection and manuscript revision.

\section{Conflicting interests: None declared.}

\section{REFERENCES}

1. Republic of Mozambique Ministry of Health (MISAU). 2018 Annual Report on HIV/AIDS Activities. MISAU; 2019. Accessed February 16, 2021. https://www.misau.gov.mz/index.php/relatorios-anuais

2. Joint United Nations Programme on HIV/AIDS (UNAIDS) AIDS Data. UNAIDS; 2016. Accessed February 11, 2021. https://www. unaids.org/sites/default/files/media_asset/2016-AIDS-data_en.pdf

3. Jani IV, De Schacht C. Innovations and challenges in early infant diagnosis of HIV. Curr Opin HIV AIDS. 2019;14(1):55-59. CrossRef. Medline

4. Violari A, Cotton MF, Gibb DM, et al.; CHER Study Team. Early antiretroviral therapy and mortality among HIV-infected infants. N Engl J Med. 2008;359(21):2233-2244. CrossRef. Medline

5. Bianchi F, Cohn J, Sacks E, et al.; EGPAF POC EID Study Team. Evaluation of a routine point-of-care intervention for early infant diagnosis of HIV: an observational study in eight African countries. Lancet HIV. 2019;6(6):e373-e381. CrossRef. Medline

6. Frank SC, Cohn J, Dunning L, et al. Clinical effect and costeffectiveness of incorporation of point-of-care assays into early infant HIV diagnosis programmes in Zimbabwe: a modelling study. Lancet HIV. 2019;6(3):e182-e190. CrossRef. Medline

7. Mwenda R, Fong Y, Magombo T, et al. Significant patient impact observed upon implementation of point-of-care early infant diagnosis technologies in an observational study in Malawi. Clin Infect Dis. 2018;67(5):701-707. CrossRef. Medline

8. Spooner E, Govender K, Reddy T, et al. Point-of-care HIV testing best practice for early infant diagnosis: an implementation study. BMC Public Health. 2019;19(1):731. CrossRef. Medline

9. Jani IV, Meggi B, Loquiha O, et al. Effect of point-of-care early infant diagnosis on antiretroviral therapy initiation and retention of patients. AIDS. 2018;32(11):1453-1463. CrossRef. Medline

10. United Nations Children's Fund (UNICEF). Key Considerations for Introducing New HIV Point-of-Care Diagnostic Technologies in National Health Systems. UNICEF; 2018. Accessed February 11 2021. https://www.who.int/hiv/pub/vct/point-of-care-hivdiagnostic/en/

11. World Health Organization (WHO). HIV Diagnosis and ARV Use in HIV-Exposed Infants: A Programmatic Update. WHO; 2018. Accessed February 11, 2021. http://apps.who.int/iris/bitstream/ handle/10665/273155/WHO-CDS-HIV-18.17-eng.pdf

12. Mozambique EID Web Portal. Instituto Nacional de Saúde. Accessed February 1 1, 2021. https://www.portaldpi.com/pentaho/Login

13. Nhabomba $C$, Chicumbe $S, M u q u i n g u e ~ H$, et al. Clinical and operational factors associated with low pediatric inpatient HIV testing coverage in Mozambique. Public Health Action. 2019;9(3):113119. CrossRef. Medline
14. Kankasa C, Carter RJ, Briggs N, et al. Routine offering of HIV testing to hospitalized pediatric patients at university teaching hospital, Lusaka, Zambia: acceptability and feasibility. J Acquir Immune Defic Syndr. 2009;51(2):202-208. CrossRef. Medline

15. McCollum ED, Preidis GA, Golitko CL, et al. Routine inpatient human immunodeficiency virus testing system increases access to pediatric human immunodeficiency virus care in sub-Saharan Africa. Pediatr Infect Dis J. 2011 ; 30(5):e75-e81. CrossRef. Medline

16. Mutanga JN, Raymond J, Towle MS, et al. Institutionalizing provider initiated HIV testing and counselling for children: an observational case study from Zambia. PLoS One. 2012;7(4):e29656. CrossRef. Medline

17. Wanyenze RK, Nawavvu C, Ouma J, Namale A, Colebunders R, Kamya MR. Provider-initiated HIV testing for paediatric inpatients and their caretakers is feasible and acceptable. Trop Med Int Health. 2010;15(1):113-119. CrossRef. Medline

18. Republic of Mozambique Ministry of Health (MISAU). National Guidelines for Implementation of Counseling and Testing. MISAU; 2015.

19. Instituto Nacional de Estatística (INE), Instituto Nacional de Saúde (INS). Study of Immunization, Malaria, and HIV/AIDS in Mozambique (IMASIDA) 2015: Report on Basic HIV Indicators. INE INS; 2018. Accessed February 16, 2021. https://dhsprogram. com/pubs/pdf/AIS12/AIS12.pdf

20. Ojikutu $B$, Higgins-Biddle $M$, Greeson $D$, et al. The association between quality of HIV care, loss to follow-up and mortality in pediatric and adolescent patients receiving antiretroviral therapy in Nigeria. PLoS One. 2014;9(7):e100039. CrossRef. Medline

21. Urick B, Fong $Y$, Okiira $C$, et al. Rapid serological tests ineffectively screen for HIV exposure in HIV-positive infants. J Acquir Immune Defic Syndr. 2018;77(3):331-336. CrossRef. Medline

22. Wagner AD, Niuguna IN, Andere RA, et al. Infant/child rapid serology tests fail to reliably assess HIV exposure among sick hospitalized infants. AIDS. 2017;31(11):F1-F7. CrossRef. Medline

23. De Schacht C, Hoffman HJ, Mabunda N, et al. High rates of HIV seroconversion in pregnant women and low reported levels of HIV testing among male partners in Southern Mozambique: results from a mixed methods study. PLoS One. 2014;9(12):e1 15014. CrossRef. Medline

24. De Schacht C, Mabunda N, Ferreira OC Jr, et al. High HIV incidence in the postpartum period sustains vertical transmission in settings with generalized epidemics: a cohort study in Southern Mozambique. J In AIDS Soc. 2014;17(1):18808. CrossRef. Medline

25. Mabunda NMC, Mutsaka D, Loquiha $O$, Buck WC, Nhachigule $C$ Meggi B. Positivity trends among HIV-exposed children tested on point-of-care early infant diagnosis in Mozambique. Poster presented at: IAS 2019; 2019; Mexico City, Mexico.

26. Republic of Mozambique Ministry of Health (MISAU). 2019 Annual Report on HIV/AIDS Activities. MISAU; 2020. Accessed February 16, 2021. https://www.misau.gov.mz/index.php/relatoriosanuais?download=367: relatorio-anual-hiv-2019-final-pdf

27. Njuguna IN, Cranmer LM, Otieno VO, et al. Urgent versus poststabilisation antiretroviral treatment in hospitalised HIV-infected children in Kenya (PUSH): a randomised controlled trial. Lancet HIV. 2018;5(1):e12-e22. CrossRef. Medline

\section{Peer Reviewed}

Received: October 8, 2020; Accepted: February 2, 2021; First published online: March 8, 2021.

Cite this article as: Matsinhe M, Bollinger T, Lee N, et al. Inpatient point-of-care HIV early infant diagnosis in Mozambique to improve case identification and linkage to antiretroviral therapy. Glob Health Sci Pract. 2021;9(1):31-39. https://doi.org/10.9745/GHSP-D-20-0061 1

(C) Matsinhe et al. This is an open-access article distributed under the terms of the Creative Commons Attribution 4.0 International License (CC BY 4.0), which permits unrestricted use, distribution, and reproduction in any medium, provided the original author and source are properly cited. To view a copy of the license, visit https://creativecommons.org/licenses/by/4.0/. When linking to this article, please use the following permanent link: https:// doi.org/10.9745/GHSP-D-20-0061 1 